\title{
A comparative study of Faine's criteria, modified Faine's criteria, and microscopic serum agglutination test to diagnose the leptospirosis
}

\author{
Parthiban Balakrishnan ${ }^{1}$, Ramkumar Padmanabhan², Sekar Pasupathy ${ }^{3}$, Balasubramanian \\ Sankaranarayanan ${ }^{4}$
}

From ${ }^{1}$ Senior Resident, ${ }^{2}$ Associate Professor, ${ }^{3}$ Professor, ${ }^{4}$ Senior Assistant Professor, Department of Pediatrics, Institute of Child Health, Madras Medical College, Chennai, Tamil Nadu, India

Correspondence to: Dr. Ramkumar Padmanabhan, Department of Pediatrics, Institute of Child Health, Madras Medical College, 7 , Daran Singh Colony, Second Street, Vadapalani, Chennai-600 026, Tamil Nadu, India. E-mail: mybabydoctor@hotmail.com

Received - 31 May 2019

Initial Review - 17 June 2019

Accepted - 20 June 2019

\begin{abstract}
Introduction: Leptospirosis is an important zoonotic infectious disease globally, caused by spirochetes from the genus Leptospira. The usefulness of Faine's criteria and modified WHO Faine's criteria 2012 for diagnosing human leptospirosis has turned out to be a valuable tool; however, limited studies have demonstrated the usefulness of these diagnostic methods in children. Objective: The objective of the study was to assess the performance characters of Faine's and modified Faine's criteria in diagnosing pediatric leptospirosis. Methods: This descriptive prospective observational study was done over a period of 2 years on children from 1 year to 12 years of age hospitalized for fever of more than 7 days duration with symptoms and signs suggestive of leptospirosis. Faine's and modified Faine's criteria were used in the diagnosis of leptospirosis, and the entire study population was tested by microscopic agglutination test (MAT). Results: A total of 91 children were included in the present study with the mean age of 5.4 \pm 1.41 years; out of which, 57 were boys and 34 were girls. All had a fever of $>39^{\circ} \mathrm{C}$ with myalgia (76\%) and headache (69\%). History of contact with the contaminated environment was present in $79 \%$ cases and $53 \%$ of cases had contact with animals. Out of 23 children diagnosed with leptospirosis using Faine's criteria, 2 were MAT positive. Out of 68 children, who were not diagnosed with Faine's criteria, 52 were MAT negative. Thirty-nine children were diagnosed by modified Faine's criteria and 17 of them were MAT positive. Fifty-two children ruled out leptospirosis using modified Faine's criteria; out of which, 51 children were MAT negative which was statistically significant. The sensitivity of $94.4 \%$ and specificity of $69.9 \%$ of modified Faine's criteria to diagnosis leptospirosis were significantly higher than the sensitivity of $11.1 \%$ and specificity of $71.2 \%$ of Faine's criteria. Conclusion: The modified Faine's criteria potentially provide a better alternative to Faine's criteria in the diagnosis of leptospirosis. Modified Faine's criteria can be very useful to diagnose leptospirosis in the resource-poor settings where the gold standard tests to diagnose leptospirosis such as culture and MAT are not available.
\end{abstract}

Key words: Enzyme-linked immunosorbent assay, Faine's criteria, Leptospirosis, Microscopic agglutination test, Serological test

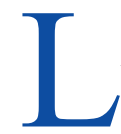

eptospirosis is a zoonotic disease of global significance with the incidence ranges from approximately $0.1-$ $1 / 100,000$ year in temperate climates to $10-100 / 100,000$ in the humid tropics $[1,2]$. The incidence rate in India is $50 / 100,000 /$ year. The WHO outbreak verification list reported an increase in the cases of leptospirosis in the state of Kerala, India, after the monsoon rains with 616 cases with 53 deaths [3]. Annually 58,900 deaths are due to several cases of leptospirosis worldwide [4]. The mortality rate in the cases of severe leptospirosis is in the range of $5-40 \%$. The mild form $(90 \%)$ of the illness is rarely fatal [5]. Wide varieties of serological tests are available with varying sensitivity and specificity. Enzyme-linked immunosorbent assay (ELISA), immunoglobulin M (IgM), IgM-specific dot-ELISA, LEPTODIPSTICK, slide agglutination method, Dri-Dot assay, complement fixation assay, latex agglutination, indirect hemagglutination test, and indirect immunofluorescence test are to name a few [6].
Faine's criteria were recommended by the world health organization as a useful tool to diagnose leptospirosis [7]. However, there is a paucity of data in the literature regarding the usefulness of Faine's criteria in pediatrics [8]. The diagnosis is established less frequently by isolation of the infecting organism from clinical specimens by culture or polymerase chain reaction during the $1^{\text {st }}$ week of illness. Serologic tests for Leptospira include genusspecific and serogroup-specific tests [9]. The reference method is the microscopic agglutination test (MAT) [10]. The drawback is that diagnosis is difficult to confirm since leptospirosis may be confused with other diseases and the disease may be mild and not be investigated in the laboratory or laboratory tests may not be available or the available tests have low sensitivity during the early phase of disease as these tests detect antibodies [11]. Hence, the present study aimed to assess the usefulness of Faine's and modified Faine's criteria in the diagnosis of leptospirosis in children. 


\section{METHODS}

The present study was a descriptive observational study which was conducted for 2 years on children from 1 year to 12 years of age hospitalized in a tertiary care hospital. The inclusion criteria consisted of children with fever lasting for more than 7 days of duration and feature suggestive of leptospirosis, including headache, myalgia, conjunctival suffusion, meningitis, and jaundice. Fever with obvious foci such as abscess, cellulitis, lymphangitis, or any child with a confirmed diagnosis other than leptospirosis at the time of discharge was excluded from the study. The sample size was calculated on the basis of the expected proportion of $21 \%$ with a confidence interval of $84 \%$ and alpha error 0.05 and calculated as 91 , with variation $40 \%$.

Children satisfying the inclusion criteria were subjected to a detailed history and thorough physical examination by a single observer. Faine's and modified Faine's scoring were carried out at the time of admission. Pro forma was filled up. $3 \mathrm{ml}$ of the blood sample was obtained by clean venipuncture with asepsis in a sterile syringe and MAT was carried out, apart from the routine investigations. Blood obtained by venipuncture was allowed to clot at room temperature, centrifuged and then MAT was carried out in (Panbio-kit) with nine live culture antigens (icterohemorrhagiae, Australis, Autumnalis, Hebdomads, Grippotyphosa, Canicola, Pomona, Patoc, and Batavia) using standard microtiter methodology.

The sera were initially screened at dilutions of 1:20 and those that were positive were titrated further to the endpoint. The highest dilution of serum that agglutinated $50 \%$ of leptospirae under dark field microscopy was presumed to represent the titer of antibody specific for the particular serogroup used. When two or more serogroups reacted at the same (highest) titer, the result was recorded as mixed-equal. Controls were put up for each one of the batteries of antigens used in the test. An initial titer of greater than or equal to $1: 160$ or a four-fold rise in titer of MAT was considered significant for the diagnosis of Leptospira infection.

The other tests performed were complete blood count, ELISA-dengue, Widal test, liver function tests, electrolytes, urea, creatinine, blood-enteric and non-enteric culture, chest X-ray, ultrasound abdomen, and CSF analysis (for meningeal signs). The second sample of blood was taken after 2 weeks of the $1^{\text {st }}$ sample for paired sera analysis of MAT either before discharge or at the review.

\section{Recommended Case Definition}

Suspected leptospirosis is a case compatible with the clinical description and presumptive laboratory diagnosis. Confirmed leptospirosis is a suspected case with confirmatory laboratory diagnosis [12].

\section{Faine's Criteria}

Faine's criteria are having Part-A with clinical data, Part-B with epidemiological factors, and Part- $\mathrm{C}$ with bacteriological lab findings with specified scores for each parameter. If the patient's score is $>25$, then leptospirosis can be diagnosed as shown in Table 1.

\section{Modified Faine's Criteria}

Certain modifications have been made in the epidemiological (Part B) and the laboratory criteria (Part C) of the original Faine's criteria. Amendments were done in 2012 to make the diagnosis more practical [13]. In the modified Faine's criteria rapid tests (ELISA/Macroscopic slide agglutination test [MSAT]) have been introduced in Part $\mathrm{C}$ and rainfall has been included in Part B to make the diagnosis early and simple to promptly start the treatment earlier [14].

\section{Statistical Analysis}

Proportion, mean and standard deviation (Mean \pm SD) of the outcome variables as applicable were collected and analyzed. Faine's criteria for the diagnosis of leptospirosis were assessed by comparing the results with the gold standard of MAT by arriving at sensitivity, specificity, positive predictive value (PPV), negative predictive value (NPV), and overall accuracy.

\section{RESULTS}

Out of the total of 91 children, who fulfilled the inclusion criteria, 57 children were male and 34 were female and maximum cases reported were between 2 and 6 years with a mean age of $5.41 \pm 1.41$ years. All had a fever with a temperature of $>39^{\circ} \mathrm{C}$ followed by myalgia (76\%) and headache (69\%). About $79 \%$ of the

Table 1: Faine's criteria

\begin{tabular}{lc}
\hline Part-A clinical data & Score \\
\hline Headache & 2 \\
Fever & 2 \\
Fever >39 & 2 \\
Conjunctival suffusion & 4 \\
Myalgia & 4 \\
Meningism & 4 \\
Abdomen pain or diarrhea & 4 \\
Conjunctival suffusion+myalgia+meningism & 10 \\
Jaundice & 1 \\
Albuminuria/nitrogen retention & 2 \\
\hline Part-B epidemiological data & Score \\
\hline Contact with animals, or contact with contaminated water & 10 \\
\hline Part-C bacterial laboratory findings & Score \\
\hline Leptospirosis endemic-positive serology & 2 \\
Single positive-low titer & 10 \\
Single positive-high titer & 25 \\
Paired sera-rising titer & 5 \\
Positive serology in leptospirosis not endemic area single & 15 \\
Positive-low titer single positive-high titer paired & 25 \\
sera-rising titer & \\
\hline
\end{tabular}


cases had a history of contact with the contaminated environment, whereas history of contact with the animals was present in 53\% cases. Validation of Faine's criteria and modified Faine's criteria with MAT is tabulated in Table 2. Out of 23 children diagnosed as leptospirosis using Faine's criteria, two were MAT positive. Out of 68 children who were leptospirosis negative utilizing Faine's criteria, 52 were MAT negative.

The performance characteristics of Faine's criteria consisted of $11 \%$ sensitivity, $71.2 \%$ specificity, $59.3 \%$ of efficacy, $8.7 \%$ of PPV, $76.5 \%$ of NPV, $28.76 \%$ of false positivity rate, and $88.88 \%$ of false negativity rate, the positive likelihood ratio was \pm 0.386 , whereas the negative likelihood ratio was \pm 1.248 . Observations from the Cohen's Kappa test were -0.160 with a slight agreement, whereas the observations from the McNemar test were 0.511, which was not significant. Validation of modified Faine's with MAT is shown in Table 2. Out of 39 children diagnosed as leptospirosis by modified Faine's criteria, 17 were MAT positive; whereas out of 52 children where leptospirosis was ruled out using modified Faine's criteria, 51 were also MAT negative, which is statistically significant.

Performance characteristics of modified Faine's criteria were as follows: Sensitivity $-94.4 \%$, specificity $-69.9 \%$, efficacy $-74.7 \%$, PPV $-43.6 \%$, NPV $-98.1 \%$, false positivity rate $-30.13 \%$, and false negativity rate $-5.55 \%$; positive likelihood ratio was 3.314, whereas the negative likelihood ratio was 0.080 . The observations from the Cohen's Kappa test were 0.447 with a moderate agreement, and the McNemar test was $<0.001$, which was highly significant.

\section{DISCUSSION}

Clinical diagnosis of leptospirosis is difficult; hence, a high index of suspicion is required for the diagnosis when a patient presents with fever, headache, and myalgia. Similar observations in the differential diagnosis of leptospirosis have been made in earlier studies [15]. A total of $18(20 \%)$ out of 91 children in the series were tested positive by MAT. A serosurvey undertaken by Ratnam et al., among conservancy workers in Chennai using MAT found a seropositive prevalence rate ranging between $17.8 \%$ and $40.5 \%$ [16].

The sensitivity of modified Faine's criteria (94.4\%) to diagnose leptospirosis was significantly higher than that of

Table 2: Validation of Faine's criteria and modified Faine's criteria with MAT

\begin{tabular}{lccc}
\hline Criteria & \multicolumn{3}{c}{ MAT } \\
\cline { 2 - 4 } & Positive & Negative & Total \\
\hline Faine's criteria & 2 & 21 & 23 \\
Positive & 16 & 52 & 68 \\
Negative & & & \\
Modified Faine's criteria & 17 & 22 & 39 \\
Positive & 1 & 51 & 52 \\
Negative & 18 & 73 & 91 \\
Total & & & \\
\hline
\end{tabular}

MAT: Microscopic agglutination test
Faine's criteria (11.1\%). The specificity of modified Faine's criteria to diagnose leptospirosis was almost equal to that of Faine's criteria (69.9\% vs. $71.2 \%)$. The sensitivity of $11.1 \%$ and specificity of $71.2 \%$ for Faine's criteria, observed in the present study, are less than that reported by Rao et al. in 2009 (88.9\% and $80.2 \%$, respectively) [17]. The contradictions of the present study with might be due to the fact that the children undertaken in the study might have not complained about occurring headache and myalgia in a similar fashion with that of adults, while the second possible scenario is conduct of the study in an endemic area in contrast to a non-endemic area where Rao and team conducted their study.

The sensitivity and specificity $(94.4 \%$ and $69.9 \%$ ) for modified Faine's criteria in the present study were similar to the observations in an earlier study [18]. The reason why modified Faine's criteria had higher sensitivity and specificity is probably that IgM Elisa and MSAT have been used in making the diagnosis of leptospirosis $[19,20]$. The efficacy of modified Faine's criteria (74.7\%) was better than that of Faine's criteria $(59.3 \%)$. When validated the Faine's criteria with the gold standard test (MAT); out of 91 patients, two were both MAT and Faine's criteria positive and while 52 were both negative. On the contrary, 17 out of 91 patients were both MAT and modified Faine's criteria positive and 51 were both negative [21].

The present study had certain limitations such as cultures which are considered as the gold standard to diagnose leptospirosis were not performed as they are very difficult to maintain, are laborious, and are time consuming [22]. As per the WHO criteria, an only presumptive diagnosis of leptospirosis was made. Cases in our series were studied only after the 5 days of the illness, and all the three serological tests (MAT, MSAT, and IgM ELISA) were positive only in about $3.29 \%$ of children. Another major limitation of the present study was the small study population.

\section{CONCLUSION}

The modified Faine's criteria can potentially provide a better diagnosis than the Faine's criteria. The modified Faine's criteria might be utilized as a diagnostic test for leptospirosis, particularly in areas of high prevalence in resource-poor developing countries where MAT cannot be done. This will enable the pediatricians to start an early treatment with specific antibiotics for the leptospirosis.

\section{REFERENCES}

1. Bharti AR, Nally JE, Ricaldi JN, Matthias MA, Diaz MM, Lovett MA, et al. Leptospirosis: A zoonotic disease of global importance. Lancet Infect Dis 2003;3:757-71

2. Human Leptospirosis: Guidance for Diagnosis, Surveillance, and Control. International Leptospirosis Society. Geneva: World Health Organization; 2003. Available from: http://whqlibdoc.who.int/hq/2003; http://www.who. int/iris/handle/10665/42667. [last accessed on 2019 May 18].

3. Izurieta R, Galwankar S, Clem A. Leptospirosis: The "mysterious" mimic. J Emerg Trauma Shock 2008;1:21-33.

4. Costa F, Hagan JE, Calcagno J, Kane M, Torgerson P, Martinez-Silveira MS, et al. Global morbidity and mortality of leptospirosis: A systematic review. PLoS Negl Trop Dis 2015;9:e0003898. 
5. Tubiana S, Mikulski M, Becam J, Lacassin F, Lefèvre P, Gourinat AC, et al. Risk factors and predictors of severe leptospirosis in new Caledonia. PLoS Negl Trop Dis 2013;7:e1991.

6. Davies D, Simonsen KA. Nelson Textbook of Pediatrics. $21^{\text {st }}$ ed. Ch. 247. Philadelphia, PA: Elsevier Publication; 2019. p. 6441-2.

7. Faine S, World Health Organization, editors. Guidelines for the Control of Leptospirosis. Geneva: World Health Organization; 1982. Available from: http://www.who.int/iris/handle/10665/37219. [last accessed on 2019 May 16].

8. Bhatia M, Kumar P, Gupta P, Gupta PK, Dhar M, Kalita D, et al. Serological evidence of human leptospirosis in patients with acute undifferentiated febrile illness from Uttarakhand, India: A pilot study. J Lab Physicians 2019;11:11-6.

9. Ahmed A, Engelberts MF, Boer KR, Ahmed N, Hartskeerl RA. Development and validation of a real-time PCR for detection of pathogenic Leptospira species in clinical materials. PLoS One 2009;4:e7093.

10. Cherry J, Demmler-Harrison G, Kaplan S, Steinbach W, Hotez P. Feigin and Cherry's Textbook of Pediatric Infectious Diseases. $8^{\text {th }}$ ed. Ch. 142. Philadelphia, PA: Elsevier Publication; 2013. p. 1263-4.

11. Desakorn V, Wuthiekanun V, Thanachartwet V, Sahassananda D, Chierakul W, Apiwattanaporn A, et al. Accuracy of a commercial IgM ELISA for the diagnosis of human leptospirosis in Thailand. Am J Trop Med Hyg 2012;86:524-7.

12. Wharton M, Chorba TL, Vogt RL, Morse DL, Buehler JW. Case definitions for public health surveillance, centre for disease control and prevention, national notifiable diseases surveillance system (NNDSS). MMWR Recomm Rep 1990;39:1-43. Available from: https://www.cdc.gov/mmwr/ preview/mmwrhtml/00025629.html. [last accessed on 2019 Mar 20].

13. Sukmark T, Lumlertgul N, Peerapornratana S, Khositrangsikun K, Tungsanga K, Sitprija V, et al. Thai-lepto-on-admission probability (THAILEPTO) score as an early tool for initial diagnosis of leptospirosis: Result from thai-lepto AKI study group. PLoS Negl Trop Dis 2018;12:e0006319.

14. Levett PN. Leptospirosis. Clin Microbiol Rev 2001;14:296-326.
15. Rajajee S, Shankar J, Dhattatri L. Pediatric presentations of leptospirosis. Indian J Pediatr 2002;69:851-3.

16. Ratnam S, Everard CO, Alex JC, Suresh B, Thangaraju P. Prevalence of leptospiral agglutinins among conservancy workers in Madras city, India. J Trop Med Hyg 1993;96:41-5.

17. Rao P, Sethi S, Sud A, Banga SS, Sharma M. Screening of patients with acute febrile illness for leptospirosis using clinical criteria and serology. Natl Med J India 2005;18:244-6.

18. Shivakumar S, Shareek PS. Diagnosis of leptospirosis utilizing modified Faine's criteria. J Assoc Physicians India 2004;52:678-9.

19. Bhatia M, Umapathy BL. Deciphering leptospirosis a diagnostic mystery: An insight. Int J Med Res Health Sci 2015;4:693-701.

20. Uy-Lumandas M, Ong-Lim AL, Gonzales ML. Validation of the modified Faine's criteria in the diagnosis of leptospirosis in children using the microscopic agglutination test as the gold standard. Pediatr Infect Dis Soc Philipp J 2013;14:42-8.

21. Bandara K, Weerasekera MM, Gunasekara C, Ranasinghe N, Marasinghe C, Fernando N, et al. Utility of modified Faine's criteria in diagnosis of leptospirosis. BMC Infect Dis 2016;16:446.

22. Budihal SV, Perwez K. Leptospirosis diagnosis: Competancy of various laboratory tests. J Clin Diagn Res 2014;8:199-202.

Funding: None; Conflict of Interest: None Stated.

How to cite this article: Balakrishnan P, Padmanabhan R, Pasupathy S, Sankaranarayanan B. A comparative study of Faine's criteria, modified Faine's criteria, and microscopic serum agglutination test to diagnose the leptospirosis. Indian J Child Health. 2019; 6(7):357-360.

Doi: 10.32677/IJCH.2019.v06.i07.007 